\title{
PrimeScreen, a brief dietary screening tool: reproducibility and comparability with both a longer food frequency questionnaire and biomarkers
}

\author{
Sheryl L Rifas-Shiman ${ }^{1}$, Walter C Willett ${ }^{2,3}$, Rebecca Lobb ${ }^{1}$, Jamie Kotch ${ }^{1}$, Charles Dart ${ }^{4}$ \\ and Matthew W Gillman ${ }^{1, *}$ \\ ${ }^{1}$ Department of Ambulatory Care and Prevention, Harvard Medical School and Harvard Pilgrim Health Care, 126 \\ Brookline Avenue, Boston, MA 02215, USA: ${ }^{2}$ Departments of Epidemiology and Nutrition, Harvard School of \\ Public Health, Boston, MA, 02115 USA: ${ }^{3}$ Channing Laboratory, Harvard Medical School and Brigham and \\ Women's Hospital, Boston, MA 02 1 15, USA: ${ }^{4}$ Harvard Center for Cancer Prevention, Harvard School of Public \\ Health, Boston, MA 02115, USA
}

Submitted 16 November 1999: Accepted 22 May 2000

\begin{abstract}
Objective: Diet is an important determinant of health outcomes, but physicians have few ways to identify persons with suboptimal diets. The purposes of this study were to examine the reproducibility of a short dietary assessment questionnaire (PrimeScreen) and to compare its results with those of a longer food frequency questionnaire and with plasma levels of selected nutrients.

Design: Each subject completed two PrimeScreen questionnaires at an interval of 2 weeks and one full length, 131-item, semiquantitative food frequency questionnaire (SFFQ), and had a sample of blood drawn. We compared the PrimeScreen with two reference standards, the SFFQ and plasma levels of selected nutrients.

Setting: A large managed care organization in New England.

Subjects: A total of 160 men and women, aged 19-65 years, participated.

Results: For foods and food groups, the mean correlation coefficient $(r)$ was 0.70 for reproducibility and 0.61 for comparability with the SFFQ. For nutrients, the mean $r$ was 0.74 for reproducibility and 0.60 for comparability with the SFFQ. No substantial differences were evident by sex, race, body mass index, occupation or education. Correlation coefficients for the comparison of vitamin E, $\beta$-carotene and lutein/ zeaxanthin intakes from the PrimeScreen with plasma levels were $0.33,0.43$ and 0.43 , respectively. These values were similar to those comparing the SFFQ with plasma levels. The median time to complete PrimeScreen was 5 min; 87\% of participants required fewer than $10 \mathrm{~min}$.

Conclusions: A quick way to assess quality of diet among adults, PrimeScreen has adequate reproducibility and its results compare well with a longer food frequency questionnaire and biomarkers.
\end{abstract}

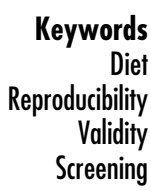

McGinnis and Foege $^{1}$ estimated that dietary factors account for at least 300000 deaths among Americans each year, making them the second leading 'actual cause' of death. Evidence exists that potentially achievable dietary changes such as increasing the daily intake of fruits and vegetables or reducing the consumption of foods high in saturated fat can have beneficial effects on long-term health ${ }^{2}$.

Physician advice/counselling can be an effective method for patient behaviour change, and thus an important contributor to public health ${ }^{3,4}$. However, a relatively small proportion of primary care physicians provide nutritional advice to their patients, owing to discomfort in giving such advice, lack of time and a perceived lack of patient interest ${ }^{5,6}$.

An additional barrier to physician involvement in dietary counselling is lack of accurate information on patients' diets. Dietary assessment tools used in research settings are generally not appropriate for routine clinical use. Multiple-day recalls and food records and even fulllength food frequency questionnaires require too much time to be practical. As a result, researchers have developed modified brief food frequency questionnaires, but generally to assess risks for only single diseases, such 
as coronary heart disease ${ }^{7-12}$, cancer $^{13-16}$ and osteoporo$\operatorname{sis}^{17,18}$, thus comprising a limited number of foods and nutrients. The shortest questionnaires to assess overall diet are at least 44 questions long ${ }^{19}$. A need exists for a brief dietary assessment tool that targets a general optimal diet for prevention of common chronic diseases of adulthood.

The purpose of this study was to assess the performance of a brief food frequency questionnaire designed to assess nutritional risk for common adult chronic diseases in the primary care setting. We examined feasibility, reproducibility and comparability with both a longer food frequency questionnaire and with plasma levels of specific nutrients.

\section{Methods}

\section{PrimeScreen questionnaire}

PrimeScreen was designed to take into account known relationships between dietary factors and major causes of morbidity and mortality in the USA, particularly cardiovascular disease, cancer and osteoporosis ${ }^{2}$. Formatted for self-completion, PrimeScreen consists of 18 questions about the average frequency of consumption, over the previous year, of specified foods and food groups, and another seven items about vitamin and supplement intake. PrimeScreen particularly targets intake of fruits, vegetables, whole and low-fat dairy products, whole grains, fish and red meat as well as other foods that are major contributors to the intake of saturated and trans fat. Within each food group, PrimeScreen includes as examples the most frequently consumed foods that constitute that group, based on data from two large cohort studies $^{20,21}$. The five frequency of consumption categories are: less than once per week, once per week, 2-4 times per week, nearly daily or daily, or twice or more per day. We compared the results of the PrimeScreen with those of the SFFQ of Willett and colleagues ${ }^{22}$. Using standard laboratory techniques ${ }^{23,24}$, we also compared reported intake of three nutrients from the PrimeScreen - vitamin E ( $\alpha$-tocopherol), $\beta$-carotene and lutein/ zeaxanthin - with plasma levels.

\section{Subjects and measurements}

Eligible patients were English-speaking men and women aged 19-65 years who were members of a large managed care organization in New England. Members were excluded if they had a diagnosis of psychosis, retardation, dementia, acquired immune deficiency syndrome (AIDS), metastatic cancer or pregnancy. We telephoned a convenience sample of 271 members with a scheduled clinical appointment, of whom 38 were not eligible: 32 individuals cancelled or changed their clinical appointment; five individuals did not speak English; and one individual was visually impaired. Of the remaining 233 individuals, 192 (82\%) agreed to participate. A total of 28 members did not keep their appointments, 164 members enrolled in the study, and four dropped out during follow-up, leaving 160 subjects who provided complete data.

At baseline, the research assistant met with the participant to obtain informed consent. We randomly assigned each subject to complete first either the PrimeScreen or the SFFQ. Both diet questionnaires were self-completed. Participants also provided sociodemographic information; we measured height and weight using a standard balance beam scale with attached stadiometer, and drew a blood sample.

At each of two follow-up timepoints, 2 and 4 weeks after baseline, each participant completed a posted selfadministered follow-up diet questionnaire. Participants who initially completed PrimeScreen received a second PrimeScreen followed by the SFFQ; those who initially completed the SFFQ received two follow-up PrimeScreens. Participants also completed an acceptance/attitudes questionnaire after the second PrimeScreen administration. Each subject received a small monetary incentive for completing the study.

\section{Data analysis}

To compare PrimeScreen with SFFQ results, we first assigned an average numerical value to each frequency category, on both questionnaires. Many questions from PrimeScreen are made up of several contributing food items. To obtain the estimated intake of each of these composite food items, we summed the weighted frequencies of each major contributing food item. We obtained weights by averaging the known frequency of consumption of each item in two large cohort studies, the Nurses Health Study (female nurses) ${ }^{20}$ and The Health Professionals Follow-up Study (male health professionals $)^{21}$. For example, the main contributors to green leafy vegetables (question one of PrimeScreen) were spinach, romaine lettuce, kale, turnip greens and bok choy. In the cohort studies, romaine lettuce contributed the largest amount (60\%) to the intake of these five foods and kale (5\%) the least.

To calculate nutrient intakes, we multiplied the weighted frequency of use (proportional to daily use $=1$ ) by the known nutrient composition of each food. We then summed the nutrients across all foods and supplements to obtain a total nutrient intake for each individual. For each supplement, we multiplied the modal dose from the large cohort studies by the frequency of consumption to obtain the nutrient intake. To obtain estimates of nutrients, we used the Harvard nutrient composition database $\mathrm{e}^{22}$.

To assess reproducibility, we compared results between the two administered PrimeScreens by computing Spearman correlation coefficients $(r)$ for each food and food group, and for 13 selected nutrients. We used two different methods to address the validity of 
Table 1 Spearman correlation coefficients $(r)$ for reproducibility and comparability with the SFFQ for PrimeScreen, a short dietary screening tool, for foods and food groups. Data from 160 men and women, aged $19-65$ years

\begin{tabular}{lcc}
\hline Foods and food groups & Reproducibility & Comparability \\
\hline Dark green leafy vegetables & 0.60 & 0.44 \\
Cruciferous veg's (broccoli, etc.) & 0.72 & 0.64 \\
Carrots & 0.76 & 0.70 \\
Other vegetables & 0.50 & 0.36 \\
Citrus fruits & 0.71 & 0.61 \\
Other fruits & 0.68 & 0.58 \\
Whole milk dairy foods & 0.59 & 0.58 \\
Low-fat dairy foods & 0.79 & 0.71 \\
Whole eggs & 0.81 & 0.82 \\
Stick margarine & 0.70 & 0.67 \\
Whole grain foods & 0.64 & 0.51 \\
Pasta, rice, noodles & 0.59 & 0.51 \\
Baked products (doughnuts, etc.) & 0.60 & 0.48 \\
Beef, pork or lamb (main dish) & 0.78 & 0.63 \\
Processed meats & 0.77 & 0.74 \\
Fish/seafood (not fried) & 0.75 & 0.57 \\
Deep fried foods & 0.77 & 0.69 \\
Added salt & 0.87 & 0.75 \\
Mean & & \\
\hline
\end{tabular}

PrimeScreen. Our primary method was to compute $r$ for the comparison of SFFQ results with the PrimeScreen administered closest in time to the SFFQ. Because of the potential for correlated error in that approach, we also computed Pearson correlation coefficients for the comparison of plasma levels with three selected nutrients from the PrimeScreen.

To assess the ability of PrimeScreen to predict individuals' intake of fruits and vegetables and per cent of energy from saturated fat, we calculated sensitivity, specificity, and positive and negative predictive values for selected cut-off points on the PrimeScreen, using the SFFQ estimates as the reference standard.

Table 2 Spearman correlation coefficients $(r)$ for reproducibility and comparability with the SFFQ for PrimeScreen, a short dietary screening tool, for selected nutrients. Data from 160 men and women, aged $19-65$ years

\begin{tabular}{|c|c|c|}
\hline Nutrients & Reproducibility & Comparability \\
\hline Beta-carotene* & 0.75 & 0.59 \\
\hline Vitamin $C^{*}$ & 0.81 & 0.71 \\
\hline Calcium* & 0.70 & 0.58 \\
\hline Iron* & 0.60 & 0.43 \\
\hline Vitamin $E^{\star}$ & 0.85 & 0.74 \\
\hline Vitamin A (retinal equivalents) ${ }^{\star}$ & 0.86 & 0.70 \\
\hline Lutein/zeaxanthin & 0.59 & 0.53 \\
\hline Animal fat & 0.75 & 0.55 \\
\hline Fibre & 0.68 & 0.58 \\
\hline Folate & 0.69 & 0.48 \\
\hline Saturated fat & 0.76 & 0.59 \\
\hline Trans fat & 0.80 & 0.64 \\
\hline Cholesterol & 0.77 & 0.63 \\
\hline Mean & 0.74 & 0.60 \\
\hline
\end{tabular}

* Includes intake from supplements as well as foods.

\section{Results}

Of the 160 participants, 57\% were females. The mean (range) age was 48 (19-65) years, and the racial/ethnic distribution was 63\% white, 31\% black, 4\% Hispanic, 1\% Asian and $1 \%$ other. A majority (59\%) held executive or professional jobs, and 59\% had graduated from college. The mean (range) body mass indexes were 27.2 (15.5$57.7) \mathrm{kg} \mathrm{m}^{-2}$ for women and 27.5 (20.1-41.1) $\mathrm{kg} \mathrm{m}^{-2}$ for men. Age, sex and race of non-participants and subjects who dropped out before completion were similar to participants.

For foods, correlation coefficients to assess reproducibility ranged from 0.50 for 'other vegetables' to 0.87 for added salt (Table 1). Correlation coefficients for comparability with the SFFQ ranged from 0.36 for 'other vegetables' to 0.82 for whole eggs. For nutrients, reproducibility ranged from 0.59 for lutein/zeaxanthin to 0.86 for vitamin A with supplements (Table 2). Comparability coefficients ranged from 0.43 for iron with supplements to 0.74 for vitamin E with supplements.

To examine the reproducibility and comparability with the SFFQ within subgroups, we stratified the population by gender, race (white and black), body mass index, education and occupation. We also examined results by random allocation to PrimeScreen or SFFQ first. No substantial differences across strata were evident (Table 3).

Correlation coefficients for the comparisons of vitamin E, $\beta$-carotene and lutein/zeaxanthin intakes from the PrimeScreen with plasma levels were $0.33,0.43$ and 0.43 , respectively. The corresponding values for comparing intake from the SFFQ with plasma levels were 0.19, 0.43 and 0.34 , respectively. Adjustment of the vitamin E levels for total plasma cholesterol level in a subset of 54 participants did not materially change the correlation with the PrimeScreen estimate of vitamin E intake $(r=0.32)$.

Table 4 shows the sensitivity, specificity, and positive and negative predictive values of Primescreen for selected cut-points for fruit and vegetable consumption and per cent of energy intake from saturated fat. The median number of servings per day of fruits and vegetables was 2.9 on PrimeScreen and 5.1 on the SFFQ. The median per cent of energy from saturated fat was 10.7 on PrimeScreen and 9.5 on the SFFQ. If an individual's intake of fruits and vegetables was less than three servings per day, as estimated by the PrimeScreen, there would be a $67 \%$ probability of 'truly' consuming less than five servings per day (positive predictive value). A PrimeScreen estimate exceeding three servings per day was associated with a $73 \%$ chance of 'truly' consuming at least five servings per day (negative predictive value). At this cut-point, $73 \%$ of individuals with 'true' intakes of less than five servings per day would be detected (sensitivity). With the PrimeScreen cut-point of $10 \%$ of energy from saturated fat, the positive predictive value would be $66 \%$ and the negative predictive value would be $80 \%$. 
Table 3 Reproducibility and comparability with the SFFQ (mean Spearman $r$ ) for PrimeScreen, a short dietary screening tool, for different subgroups. Data from 160 men and women, aged 19-65 years

\begin{tabular}{|c|c|c|c|c|c|c|c|c|c|c|c|c|}
\hline & \multicolumn{2}{|c|}{ Sex } & \multicolumn{2}{|c|}{ Race $^{*}$} & \multicolumn{2}{|c|}{ BMI } & \multicolumn{2}{|c|}{ Occupation } & \multicolumn{2}{|c|}{ Education } & \multicolumn{2}{|c|}{ Assignment } \\
\hline & $\begin{array}{c}\text { Female } \\
(n=91)\end{array}$ & $\begin{array}{c}\text { Male } \\
(n=69)\end{array}$ & $\begin{array}{l}\text { White } \\
(n=101)\end{array}$ & $\begin{array}{c}\text { Black } \\
(n=49)\end{array}$ & $\begin{array}{c}\text { BMl } \\
\leq 27 \mathrm{~kg} \mathrm{~m}^{-2} \\
(n=91)\end{array}$ & $\begin{array}{c}\mathrm{BMI} \\
>27 \mathrm{~kg} \mathrm{~m}^{-2} \\
(n=68)\end{array}$ & $\begin{array}{l}\text { Executive/ } \\
\text { professional } \\
(n=94)\end{array}$ & $\begin{array}{c}\text { Other } \\
(n=66)\end{array}$ & $\begin{array}{c}\text { College } \\
\text { graduate } \\
(n=95)\end{array}$ & $\begin{array}{c}\text { Some/no } \\
\text { college } \\
(n=65)\end{array}$ & $\begin{array}{c}\text { FPP } \\
(n=82)\end{array}$ & $\begin{array}{c}\text { PPF } \\
(n=78)\end{array}$ \\
\hline \multicolumn{13}{|c|}{ Foods and food groups } \\
\hline Reproducibility & 0.69 & 0.70 & 0.72 & 0.64 & 0.71 & 0.67 & 0.74 & 0.65 & 0.74 & 0.64 & 0.74 & 0.67 \\
\hline Comparability & 0.56 & 0.61 & 0.60 & 0.58 & 0.61 & 0.61 & 0.63 & 0.57 & 0.63 & 0.57 & 0.63 & 0.59 \\
\hline \multicolumn{13}{|l|}{ Nutrients } \\
\hline Reproducibility & 0.72 & 0.76 & 0.78 & 0.72 & 0.75 & 0.72 & 0.77 & 0.71 & 0.78 & 0.69 & 0.77 & 0.73 \\
\hline Comparability & 0.56 & 0.62 & 0.63 & 0.52 & 0.57 & 0.62 & 0.65 & 0.54 & 0.64 & 0.54 & 0.57 & 0.61 \\
\hline
\end{tabular}

BMI, body mass index; FPP, random allocation to the SFFQ followed by two PrimeScreens; PPF, random allocation to two PrimeScreens followed by the SFFQ.

* Race analysis limited to white and African-American (black) subjects only.

Based on the results of the follow-up attitude survey, over $90 \%$ of participants found PrimeScreen easy or very easy to understand and complete. The median time to complete PrimeScreen was $5 \mathrm{~min} ; 87 \%$ of participants required fewer than $10 \mathrm{~min}$.

\section{Discussion}

Our findings suggest that PrimeScreen has adequate reproducibility and ranks individuals reasonably well by intake of foods and nutrients. The correlation coefficients for comparability with the SFFQ, ranging from approximately 0.4 to 0.8 , compare favourably with those of other brief questionnaires designed to assess a more limited range of foods and nutrients relevant to specific disease conditions $^{7,9-14,16-18}$. For example, investigators using short questionnaires designed to assess cardiac risk have examined their accuracy in measuring saturated fat intake. Compared with diet recalls or records, correlation coefficients ranged from 0.30 to $0.58^{9,11,13}$; our estimate was 0.59 .
In two studies that examined fibre intake, the correlations were $0.57^{11}$ and $0.50^{16}$, close to our value of 0.58 . Cummings et $\mathrm{al}^{17}$ examined the validity of a five-item, a 10 -item and a 15-item questionnaire designed to assess calcium intake. Validity estimates were $0.67,0.75$ and 0.76 , respectively. For PrimeScreen, which had only two questions reflecting calcium intake, the correlation coefficient for comparability with the SFFQ was 0.58 .

Substantial correlations between nutrient intakes from PrimeScreen and plasma levels provided additional documentation of validity. They are especially useful because measurement errors in the plasma markers are likely to be uncorrelated with errors in the PrimeScreen. The estimates for the three selected nutrients were similar to those comparing the SFFQ with plasma levels, both in our study and in previous validation studies of the SFFQ $^{26,27}$.

All food frequency type questionnaires require memory and averaging skills. Thus, it is not surprising that PrimeScreen performed better in assessing single-food

Table 4 Ability of PrimeScreen to predict individuals' intake of fruits and vegetables and saturated fat. Comparison with SFFQ among 160 men and women, aged $19-65$ years*

\begin{tabular}{|c|c|c|c|c|}
\hline PrimeScreen cut-point & Sensitivity & Specificity & Positive predictive value† & Negative predictive valuef \\
\hline \multicolumn{5}{|c|}{ Fruits and vegetables ( $<5$ servings day ${ }^{-1}$ ) } \\
\hline 2.0 & 0.42 & 0.94 & 0.86 & 0.63 \\
\hline 3.0 & 0.73 & 0.67 & 0.67 & 0.73 \\
\hline 4.0 & 0.92 & 0.45 & 0.61 & 0.86 \\
\hline 5.0 & 0.96 & 0.23 & 0.54 & 0.86 \\
\hline \multicolumn{5}{|c|}{ Saturated fat ( $\geq 10 \%$ energy) } \\
\hline 6 & 0.99 & 0.39 & 0.58 & 0.97 \\
\hline 8 & 0.92 & 0.55 & 0.63 & 0.89 \\
\hline 10 & 0.81 & 0.66 & 0.66 & 0.80 \\
\hline 12 & 0.60 & 0.79 & 0.71 & 0.70 \\
\hline 14 & 0.48 & 0.91 & 0.81 & 0.68 \\
\hline
\end{tabular}

* In this population, based on the SFFQ, $48 \%$ consumed $<5$ servings of fruits and vegetables per day and $46 \%$ consumed $\geq 10 \%$ energy from saturated fat. † Positive predictive value means the probability that a person 'truly' consumes $<5$ servings of fruits and vegetables or $\geq 10 \%$ energy from saturated fat, given that he or she tests positive (individual consumes less than the cut-point for fruits and vegetables or more than the cut-point for energy from saturated fat), as estimated from the PrimeScreen.

$\ddagger$ Negative predictive value means the probability that a person 'truly' consumes $\geq 5$ servings of fruits and vegetables or $<10 \%$ energy from saturated fat, given a negative screening test (individual consumes more than the cut-point for fruits and vegetables or less than the cut-point for energy from saturated fat), as estimated from the PrimeScreen. 
items than multi-item food groups, which required individuals to consider many contributors. For example, the correlation coefficients for comparability with the SFFQ for whole eggs and carrots were 0.82 and 0.70 , respectively. By contrast, the correlation coefficients for dark green leafy vegetables and baked products were 0.44 and 0.48 , respectively.

Several attributes of this study strengthen confidence in the results. Correlations did not vary appreciably by random assignment, suggesting that the process of completing the SFFQ did not affect response to PrimeScreen. Also, results comparing the SFFQ to the more temporally distant, rather than the closer, PrimeScreen gave similar results (data not shown). The study sample was diverse in terms of race, age and sex, and the results did not vary by these factors. There were no substantial differences across strata of education and occupation, but the study participants had relatively high educational status and most had executive/professional occupations. It is possible that results would differ in socioeconomic strata, ethnic groups and geographic regions not represented in the study sample. Thus it would be useful to measure the performance of PrimeScreen in different populations.

One limitation is that PrimeScreen was not designed to assess the total diet, and therefore cannot measure total energy intake. This factor limits our ability to estimate absolute nutrient intakes and compare them with existing standards, such as recommended daily allowances. To avoid bias due to correlated error with the use of two like instruments, food records or recalls are often used as the reference standard in validation studies of food frequency questionnaires. Unfortunately, food records or recalls were not feasible in this study. Food frequency questionnaires, however, can serve as surrogate reference standards since they have been used to detect numerous diet-disease relationships in several large epidemiological studies ${ }^{20,21,25}$. One other study has used a full-length SFFQ as a reference standard ${ }^{12}$. In that study, in which the investigators used an 18-item questionnaire to determine heart disease risk, the correlation for total fat as a per cent of energy, the only nutrient correlation reported, was 0.47 , in the same range as other studies using food records as standards for comparison ${ }^{10,13}$.

PrimeScreen is a general dietary screening tool that is easy to complete and requires minimal time. Its format, focusing on commonly eaten foods and a limited number of response categories, makes it potentially suitable as an intervention tool in clinical practice as well as research. Future research should focus on finding optimal ways to use this tool in effecting dietary change among individuals and populations.

\section{Acknowledgements}

We thank the following persons for their diverse contributions to this project: Laura Sampson, Jelia Witschi RD, Thomas Hawkins MD, Xiaoming Gao MS, Cindy L Christiansen PhD, Nadine Braunstein RD, Nancy Rigotti MD and William Owusu ScD.

\section{References}

1 McGinnis JM, Foege WH. Actual causes of death in the United States. JAMA 1993; 270(18): 2207-12.

2 Willett WC. Diet and health: what should we eat? Science 1994; 264: 532-7.

3 Russel MAH, Wilson C, Taylor C, Baker CD. Effect of general practitioners' advice against smoking. BMJ 1979; 2: 231-5.

4 Kelly RB. Controlled trial of a time-efficient method of health promotion. Am. J. Prev. Med. 1988; 4: 200-7.

5 Glanz K, Gilboy MB. Physicians, preventive care, and applied nutrition: selected literature. Acad. Med. 1992; 67: 776-81.

6 Kottke TE, Foels JK, Hill C, Choi T, Fenderson DA. Nutrition counseling in private practice: Attitudes and activities of family physicians. Prev. Med. 1984; 13: 219-25.

7 Conner SL, Gustafson JR, Sexton G, Becker N, Artaud-Wild S, Conner WE. The diet habit survey: a new method of dietary assessment that relates to plasma cholesterol changes. J. Am. Diet. Assoc. 1992; 92: 41-7.

8 Knapp JA, Hazuda HP, Haffner SM, Young EA, Stern MP. A saturated fat/cholesterol avoidance scale: sex and ethnic differences in a biethnic population. J. Am. Diet. Assoc. 1988; 88: $172-7$.

9 Heller RF, Pedoe HDT, Rose G. A simple method of assessing the effect of dietary advice to reduce plasma cholesterol. Prev. Med. 1981; 10: 364-70.

10 Peters JR, Quiter ES, Brekke ML, et al. The Eating Pattern Assessment Tool: a simple instrument for assessing dietary fat and cholesterol intake. J. Am. Diet. Assoc. 1994; 94(9): 100813.

11 Ammerman AS, Haines PS, DeVellis RF, et al. A brief dietary assessment to guide cholesterol reduction in low-income individuals: design and validation. J. Am. Diet. Assoc. 1991; 91: 1385-90.

12 Lefebvre RC, Linnan L, Sundaram S, Ronan A. Counseling strategies for blood cholesterol screening programs: recommendations for practice. Patient Educ. Counsel. 1990; 16: 97-108.

13 Block G, Clifford C, Naughton MD, Henderson M, McAdams M. A brief dietary screen for high fat intake. J. Nutr. Educ. 1989; 21: 199-207.

14 Serdula M, Coates R, Byers T, et al. Evaluation of a brief telephone questionnaire to estimate fruit and vegetable consumption in diverse study populations. Epidemiology 1993; 4: 455-63.

15 Byers T, Marshall J, Fiedler R, Zielezny M, Graham S. Assessing nutrient intake with an abbreviated dietary interview. Am. J. Epidemiol. 1985; 122: 41-50.

16 Shannon J, Kristal AR, Curry SJ, Beresford SA. Application of a behavioral approach to measuring dietary change: the fatand fiber-related diet behavior questionnaire. Cancer Epidemiol. Biomarkers Prev. 1997; 6(5): 355-61.

17 Cummings SR, Block G, McHenry K, Baron RB. Evaluation of two food frequency methods of measuring dietary calcium intake. Am. J. Epidemiol. 1987; 126: 796-802.

18 Angus RM, Sambrook PN, Pocock NA, Eisman JA. A simple method for assessing calcium intake in Caucasian women. $J$. Am. Dietet. Assoc. 1989; 89(2): 209-14.

19 Pietinen P, Hartman AM, Haapa E, et al. Reproducibility and validity of dietary assessment instruments. II. A qualitative food-frequency questionnaire. Am. J. Epidemiol. 1988; 128: $667-76$. 
20 Colditz GA. The Nurses Health Study: a cohort of US women followed since 1976. J. Am. Med. Wom. Assoc. 1995; 50: 40-4.

21 Ascherio A, Rimm EB, Giovannucci EL, et al. A prospective study of nutritional factors and hypertension among US men. Circulation 1992; 86: 1475-84.

22 Rimm EB, Giovannucci EL, Stampfer MJ, Colditz GA, Litin LB, Willett WC. Reproducibility and validity of an expanded self-administered semiquantitative food frequency questionnaire among male health professionals. Am. J. Epidemiol. 1992; 135: 1114-26.

23 Bieri JG, Brown ED, Smith JC Jr. Determination of individual carotenoids in human plasma by high performance liquid chromatography. J. Liquid Chromatogr. 1985; 8: 473-84.

24 Motchnik PA, Frei B, Ames BN. Measurement of antioxidants in human blood plasma. Methods Enzymol. 1994; 234: 26979.

25 Kushi LH, Lew RA, Stare FJ, et al. Diet and 20-year mortality from coronary heart disease: The Ireland-Boston diet heart study. New Engl. J. Med. 1985; 312: 811-18.

26 Willett WC, Stampfer MJ, Underwood BA, Speizer FE, Rosner $\mathrm{B}$, Hennekens $\mathrm{CH}$. Validation of a dietary questionnaire with plasma carotenoid and $\alpha$-tocopherol levels. Am.J. Clin. Nutr. 1983; 38: 631-9.

27 Ascherio A, Stampfer MJ, Colditz GA, Rimm EB, Litin L, Willett WC. Correlations of vitamin A and $\mathrm{E}$ intakes with the plasma concentrations of carotenoids and tocopherols among American men and women. J. Nutr. 1992; 122(9): $1792-801$. 